\title{
Pruning Discrete and Semicontinuous Skeletons
}

\author{
Dominique Attali ${ }^{+}$, Gabriella Sanniti di Baja ${ }^{*}$, Edouard Thiel ${ }^{+}$
}

\author{
*Istituto di Cibernetica, CNR, Via Toiano 6, 80072 Arco Felice, Naples, Italy \\ +Equipe TIMC-IMAG, IAB-Domaine de la Merci, 38706 La Tronche cedex, France
}

\begin{abstract}
In this paper pruning techniques are illustrated, which allow us to suitably simplify the (discrete and semicontinuous) skeleton, by either deleting or shortening peripheral skeleton branches. To avoid excessive shortening, which might reduce the representative power of the skeleton, the relevance of the figure regions mapped in the skeleton branches is used to decide on pruning. Different definitions of relevance are introduced and features allowing the quantitative evaluation of the relevance are suggested.
\end{abstract}

\section{Introduction}

The skeleton is a stick-like representation of a figure, which accounts for different figure properties. It is a curvilinear set consisting of branches and, in case of multiply connected figures, of loops. Each skeleton component is placed in the medial regions of a figure subset, and is oriented along the directions of the main symmetry axes of the corresponding figure subset. Each element of the skeleton can be interpreted as the centre of a disc fitting the figure, and is labelled with the corresponding radius; thus, the length of a skeleton branch gives an evaluation of the elongation of the represented figure subset and the labels of the skeleton elements provide a measure of the local thickness of the figure.

The literature includes a relevant number of papers dealing with skeletonization. Most of them refer to the computation of discrete skeletons. More recently, algorithms using the Voronoi graph have become of interest to compute semicontinuous skeletons. In fact the computation cost of the Voronoi graph is no longer prohibitive; moreover vertices approximating the figure contour in the continuous plane are often available, which can be used directly to guide skeletonization without performing any shape digitisation. A problem affecting both discrete and continuous skeletons is the presence of a number of peripheral skeleton branches, originated in correspondence with figure protrusions having no perceptual relevance. This makes the skeleton structure complex and limits the possibility to use the skeleton for shape analysis. This paper provides different pruning criteria for the discrete and the continuous skeleton, which allow us to eliminate unwanted branches without significantly altering the topological and representative power of the skeleton.

\section{Discrete Skeleton}

On the discrete plane, the identification of the skeletal pixels can be conveniently accomplished on the distance map of the figure (e.g., $[1,2])$. The distances most commonly used to compute the distance map are the city-block distance, the chessboard distance, the 
$(3,4)$-weighted distance and the $(5,7,11)$-weighted distance. The latter two distances, introduced in [3], provide a better approximation to the Euclidean distance and allow to originate skeletons almost stable under figure rotation. On the distance map, one can identify a nearly thin set of skeletal pixels (i.e., the centres of the maximal discs, the saddle pixels, and the linking pixels). This set is then reduced to the unit wide skeleton, by employing removal operations, which are topology-and-end-point preserving.

The discrete skeleton cannot be perfectly centred within the figure, wherever the thickness of the figure is given by an even number of pixels. In these regions, the set of the centres of maximal discs is 2-pixel wide. The unit wide skeleton includes almost all the centres of the maximal discs, so that the figure can be nearly completely recovered by applying to its skeleton the reverse distance transformation. Complete recovery is not compatible with skeleton unit thickness. Each skeleton pixel is the centre of a disc fitting the shape. The discs are polygons approximating the Euclidean circle to a different extent, depending on the adopted distance function. Discs obtained by the city-block distance and chessboard distance are 4-side polygons, while those obtained via the $(3,4)$-weighted distance and the $(5,7,11)$-distance are 8-side and 16-side polygons, respectively. Maximal discs, associated with skeleton pixels sufficiently close to each other, partially overlap so that the set of the maximal discs does not provide a partition of the figure. The contour of a maximal disc and the contour of the figure share one, two or more connected subsets, each of which may include more than one pixel.

\section{Semicontinuous Skeleton}

Semicontinuous skeletonization does not require image digitisation. The skeleton is a graph, computed starting from a polygonal approximation of the continuous shape (provided, for instance, by segmentation methods using a deformable curve model). The vertices of the polygonal approximation in the continuous plane sample the boundary of the continuous shape, and are called the sampling points. A measure of the quality of the approximation is given by bounding the greatest distance between two neighbouring sampling points on the boundary. The more numerous those sampling points, the more accurate the approximation.

In recent papers [4-6], the skeleton of continuous shapes is approximated by using the Voronoi graph of the sampling points [7]. A partition of the polygonal shape is computed by using the Delaunay triangulation; then, the approximated skeleton is defined as the dual of this partition. For sufficiently regular shapes, due to the convergence theorem [8], the Voronoi vertices of the sampling points tend to the complete skeleton (i.e., the endoskeleton and the exoskeleton of the shape) when the sampling points tend to the shape boundary. The semicontinuous skeleton consists of the Voronoi vertices associated with the Delaunay triangles contained inside the shape, and of the straight line segments connecting the vertices. Two vertices are connected by a segment if their associated triangles are adjacent. To reconstruct the continuous shape, one can use either the Delaunay triangles associated with the Voronoi vertices, or the Delaunay discs (i.e., discs circumscribed to Delaunay triangles). Triangles provide a partition of the region of the plane enclosed by the initial polygonal approximation. In turn, the discs partially overlap. Their union tends to the continuous shape, when the density of the sampling points sufficiently increases.

\section{Pruning}

To avoid topology modifications, pruning always concerns with peripheral branches, i.e., branches delimited by an end point. In the discrete skeleton, an end point is a skeleton pixel 
having only one neighbour in the skeleton. In the semicontinuous case, an end point is a Voronoi vertex having only one neighbouring vertex in the skeleton. The second extreme of a skeleton branch is generally a branch point. In the discrete case, this is a pixel with more than two neighbours in the skeleton; in the continuous case it's a Voronoi vertex having exactly three neighbouring vertices in the skeleton. All the other pixels of a discrete skeleton branch (Voronoi vertices of a semicontinuous skeleton branch) have exactly two neighbours (neighbouring vertices) in the skeleton, and are called normal points. In the following, the term skeleton will be used to equivalently refer to the discrete or the continuous skeleton; the term element will denote a skeletal pixel or a Voronoi vertex.

The discrete skeleton [1] computed by using the $(5,7,11)$-weighted distance and the semicontinuous skeleton [6] of a test shape are respectively shown in Fig.1a and Fig.1b, as they result before applying any pruning. Pruning may involve either partial shortening or complete deletion of a peripheral branch. The elements of the branch are checked one after the other against a given pruning condition. Pruning is accomplished as far as the pruning condition is satisfied. Generally, the pruning condition should prevent excessive shortening of skeleton branches, as this may result in loss of skeleton representation power. Thus, pruning should be based on a measure of protrusion relevance and the only branches to be pruned are those associated with protrusions regarded as non meaningful according to the relevance measure.

When all the branches sharing a branch point are totally deleted, new peripheral branches are possibly originated in the modified skeleton. These branches can be furthermore subjected to pruning, provided that the protrusion whose relevance is evaluated is the protrusion mapped in the union of the current peripheral skeleton branch with the neighbouring, already pruned, skeleton branches.

\subsection{Pruning Criteria}

Branch length. The length of a peripheral branch can be computed in terms of the number of elements constituting the branch. A peripheral branch can be entirely removed if its length is below an a priori fixed threshold. This criterion has not general applicability, as the length of a noisy branch depends also on the thickness of the region from which the noisy protrusion sticks out. A length based criterion can be used to remove very short branches, say 1 or 2 elements, or for particular classes of figures (e.g., alphanumerics, where any figure is the superposition of elongated narrow strokes having constant thickness).

In the discrete case, the distribution and number of centres of maximal discs along the branch can provide some more information on the relevance of the represented protrusion. Generally, a small percentage of pixels of a noisy branch are centres of maximal discs. In the continuous skeleton, by using the Euler constant one can prove that the number of skeleton vertices is related to the number of sampling points. If the sampling points are regularly spaced, then, computing the number of vertices is equivalent to computing the length of the boundary of the protrusion associated with the branch. Pruning methods based on the computation of the length of the boundary have been proposed in [5].

Intuitively, a portion of a branch can be safely pruned if a negligible difference exists between the two regions corresponding to the entire skeleton branch and to the pruned skeleton branch, respectively. The difference in elongation or in area between the previous regions can be used to decide on pruning.

Elongation. Let $\mathrm{r}$ and $\mathrm{R}$ be the radil of the discs associated with the end point $\mathrm{p}$ of $\mathrm{a}$ peripheral skeleton branch and a more internal element $q$, along the same skeleton branch. Let $d$ be the distance between the two elements $p$ and $q$. The quantity $(r-R+d)$ measure the distance between the contour of the two regions, respectively associated with the entire 
(a)
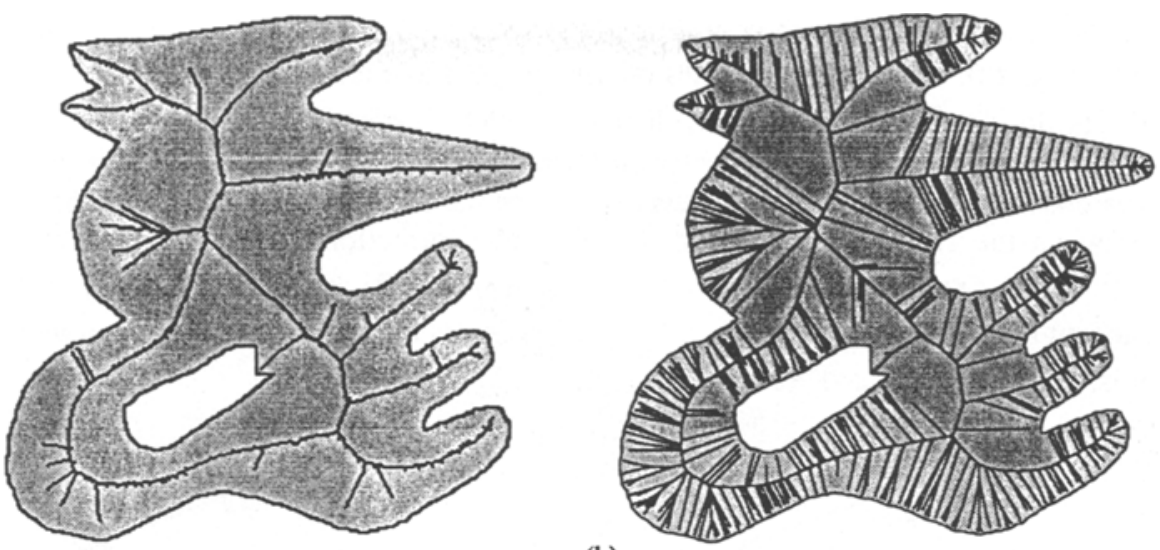

(b)

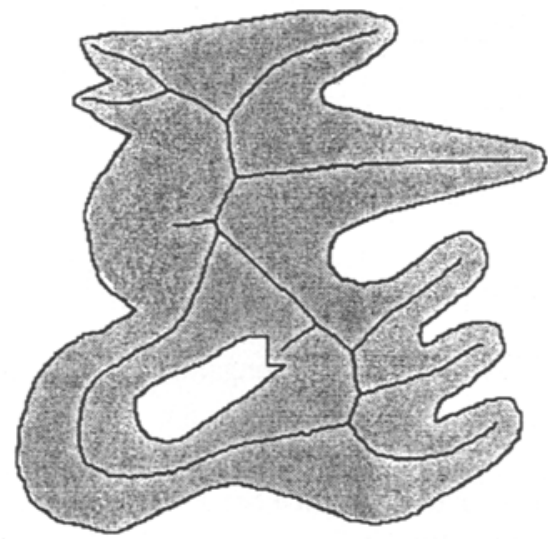

(c)

(d)
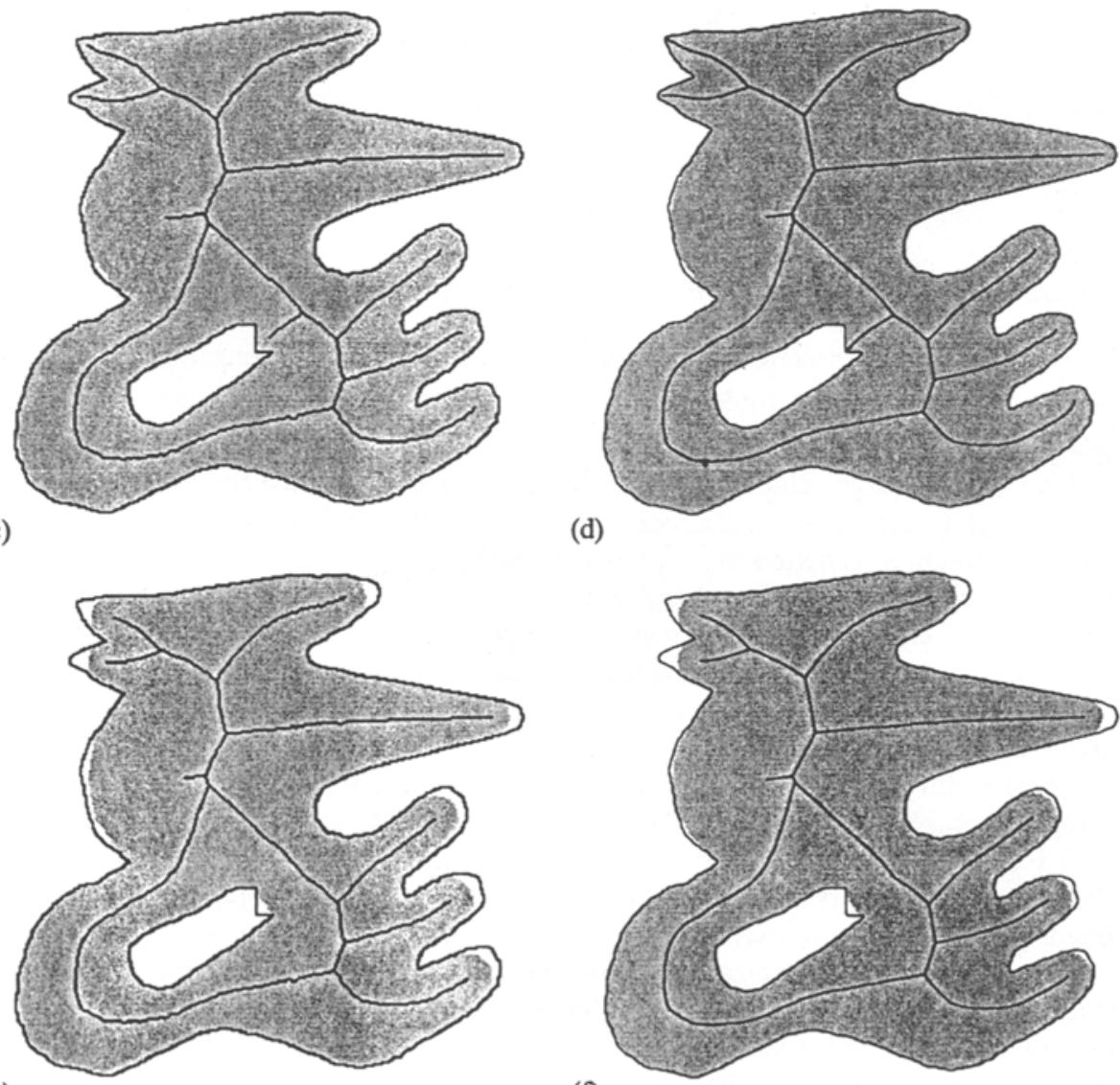

(e)

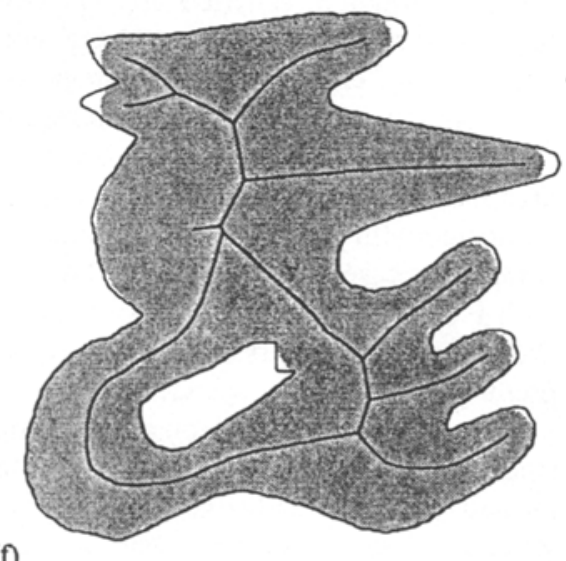

(f)

Fig. 1. The discrete skeleton, (a), and the continuous skeleton, (b), before pruning. Effect of elongation-based pruning, (c), and area-based pruning, (e), on the discrete skeleton. Effect of area-based pruning on the continuous skeleton: the protrusion area is compared with the area of a single disc in (d), and with the area of the entire shape in (f). 
branch, and with the branch pruned up to $\mathrm{q}$ ( $\mathrm{q}$ excluded). This value can be compared with a threshold $\vartheta$, whose value depends on the accepted tolerance in figure recovery. Pruning can be done up to the most internal element $q$ such that $(r-R+d) \leq \vartheta$.

A disadvantage of the above criterion is that it does not take into account protrusion sharpness. Since protrusion sharpness depends on the difference in radii and on the distance between the two elements $p$ and $q$, a suitable correction factor can be accordingly introduced. Our choice is to multiply $\vartheta$ by $(R-r+1) / d$, which approximately evaluates the tangent of the angle $\beta$, as shown in Fig.2a. Pruning is accomplished up to q, provided that it is: $(r-R+d) \leq \vartheta \times(R-r+1) / d$.

(a)

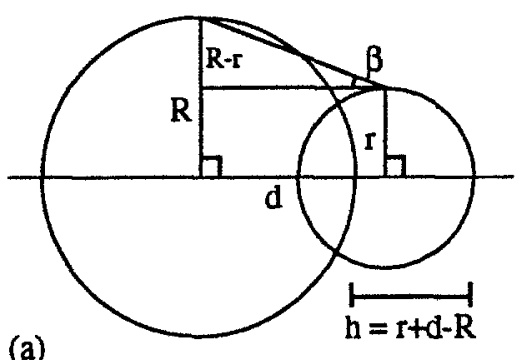

Fig. 2. (a) The angle $\beta$ changes with protrusion sharpness; (b) the area of the protrusion (dark region) can be computed in terms of the radii $R$ and $r$, and of the angle $\alpha$.

Fig.1c shows the discrete skeleton, pruned by using the above elongation-based criterion with $\vartheta=2$. White regions inside the shape contour identify the pixels non recovered by the pruned skeleton.

Note that if a large value is assigned to $\vartheta$, the geodesic distance between $p$ and $q$ should be employed in place of the distance $d$, when evaluating $(r-R+d)$. Otherwise, the pruning condition might be satisfied also by two elements $\mathrm{p}$ and $\mathrm{q}$ of a significant skeleton branch, along which relevant curvature changes occur.

Area. The difference between the region corresponding to the skeleton branch including all the elements from $p$ to $q$, and the region associated to the element $q$ alone, defines the protrusion that would be flattened by pruning the skeleton branch up to $q, q$ excluded. The area $P$ of the protrusion can be directly compared with a threshold or, preferably, it can be compared with the area $\mathrm{F}$ of the whole figure (easily available in both the discrete and the continuous case) or with the area $\mathrm{D}$ of the region associated to the element $q$. Comparing $P$ with $\mathrm{F}$ allows one to use the same threshold whichever is the size of the figure at hand. Pruning will be equally effective on equally sized protrusions. Comparing $P$ with $D$ makes pruning more context dependent.

In the continuous skeleton case, protrusion area evaluation can be accomplished easily, since the skeleton vertices are associated with non overlapping triangles. In turn, the Delaunay discs partially overlap each other and also one Delaunay disc can overlap partially several Delaunay triangles. One can make use of this remark to provide a better pruning criterion as well as a more faithful shape reconstruction. The key idea is that of using the Delaunay triangles while evaluating the contributions provided by the Voronoi vertices that are going to be removed by pruning (i.e., the vertices from $p$ to $q$, $q$ excluded), and to use the Delaunay discs when evaluating the area of the region associated to $q$ and, in general, for shape recovery. If the current protrusion mapped in the skeleton branch from $p$ to $q$ is 
significantly overlapped by the Delaunay disc associated to $q$, then the branch can be safely removed; a rather faithful recovery is still possible, provided that the shape is reconstructed by using the union of the Delaunay discs rather than the union of the triangles. Let $P$ be the area obtained by adding the area of the Delaunay triangles associated with the skeleton vertices from $p$ to $q, q$ excluded. Let $D$ be the area of the Delaunay disc associated to $q$. The branch is shortened up to $q$ if it results: $P \leq \vartheta \times D$. Alternatively, if $F$ denotes the area of the shape, pruning can be accomplished provided that $\mathrm{P} \leq \vartheta \times \mathrm{F}$. In both cases, the value of $\vartheta$ depends on the tolerance in figure recovery.

Fig. $1 \mathrm{~d}, \mathrm{f}$ shows the continuous skeleton after applying the area-based pruning; shape reconstruction is done by employing the Delaunay discs. Both the polygonal approximation of the initial shape and the reconstructed shape (grey region) are illustrated. In Fig.1d, the area $\mathrm{P}$ of the protrusion is compared with $\mathrm{D}$ and it is $\vartheta=0,25$; in Fig.1f, $\mathrm{P}$ is compared with the area of the entire shape and it is $\vartheta=0,005$.

In the discrete case, since the maximal discs partially overlap, generally the computation of the area of the protrusion is not straightforward. Only for skeletons driven by the cityblock and the chessboard distances (i.e., in case of square-shaped discs), convenient algorithms have been introduced to compute the area of the union of the maximal discs [9]. For the general case of skeletons driven by weighted distances, providing more rounded discs, an approximated evaluation of the protrusion area can be computed as $\left(\mathrm{R}^{2}-\mathrm{r}^{2}\right) \times(\tan$ $\alpha-\alpha)$, where $\alpha$ is the angle shown in Fig.2b.

As in the continuous case, the area of the protrusion can be compared with the area $\mathrm{F}$ of the whole figure, or the area $D$ of the disc associated with the element $q$. Fig.le, shows the effect of the area-based criterion on the discrete skeleton, when the area of the protrusion is compared with $F$ and the threshold is $\vartheta=0,03$. As before, both the initial shape and the reconstructed shape (grey region) are illustrated.

\section{Acknowledgements}

This work has been partially supported by the "Galileo Program for the cooperation between France and Italy".

\section{Reference}

1. G. Sanniti di Baja, E. Thiel: Computing and comparing distance-driven skeletons. In: C. Arcelli et al. (eds.): Aspects of Visual Form Processing. Singapore: World Scientific, 1994, pp. 475-486.

2. E. Thiel: Les distances de chanfrein en analyse d'images: fondements et applications. $\mathrm{PhD}$ Thesis. Grenoble 1994. University J. Fourier.

3. G. Borgefors: Distance transformation in digital images. CVGIP, 34, 344-371 (1986).

4. J.W. Brandt, V.R. Algazi: Continuous skeleton computation by Voronoi diagram. CVGIP: Image Understanding. 55, 329-337 (1992).

5. R.L. Ogniewicz: Discrete Voronoi skeletons. Konstanz: Hartung-Gorre Verlag 1993.

6. D. Attali, A. Montanvert: Semicontinuous skeletons of $2 \mathrm{D}$ and $3 \mathrm{D}$ shapes. In C.Arcelli et al. (eds.): Aspects of Visual Form Processing. Singapore: World Scientific 1994, pp. 32-41.

7. M. Schmitt: Some examples of algorithms analysis in computational geometry by means of mathematical morphology techniques. In J.D. Boissonat et al. (eds.): Geometry and Robotics. Lecture Notes in Computer Science. Berlin: Springer-Verlag 1989, pp. 225-246.

8. F.P. Preparata, M.I. Shamos: Computational Geometry: an Introduction. Text and Monographs in Computer Science. Berlin: Springer-Verlag 1988.

9. L.P. Cordella, G. Sanniti di Baja: Geometric properties of the union of maximal neighborhoods. IEEE Trans. on PAMI, 11-2, pp. 214-217 (1989). 